\title{
Excited-State Relaxation of Some Aminoquinolines
}

\author{
M. N. Khimich and B. M. Uzhinov \\ Chemistry Department, M. V. Lomonosov Moscow State University, Vorob'evy Gory, 119992 Moscow, Russia
}

Received 25 April 2005; Revised 6 July 2005; Accepted 13 July 2005

\begin{abstract}
The absorption and fluorescence spectra, fluorescence quantum yields and lifetimes, and fluorescence rate constants $\left(k_{f}\right)$ of 2 amino-3-(2'-benzoxazolyl)quinoline (I), 2-amino-3-(2'-benzothiazolyl)quinoline (II), 2-amino-3-( $2^{\prime}$-methoxybenzothiazolyl)quinoline (III), 2-amino-3-(2'-benzothiazolyl)benzoquinoline (IV) at different temperatures have been measured. The shortwavelength shift of fluorescence spectra of compounds studied $(23-49 \mathrm{~nm}$ in ethanol) as the temperature decreases (the solvent viscosity increases) points out that the excited-state relaxation process takes place. The rate of this process depends essentially on the solvent viscosity, but not the solvent polarity. The essential increasing of fluorescence rate constant $k_{f}$ (up to about 7 times) as the solvent viscosity increases proves the existence of excited-state structural relaxation consisting in the mutual internal rotation of molecular fragments of aminoquinolines studied, followed by the solvent orientational relaxation.
\end{abstract}

Copyright (c) 2006 M. N. Khimich and B. M. Uzhinov. This is an open access article distributed under the Creative Commons Attribution License, which permits unrestricted use, distribution, and reproduction in any medium, provided the original work is properly cited.

\section{INTRODUCTION}

The excited-state intramolecular proton transfer (ESIPT) reaction is one among few known primary photochemical processes which result in the appearance of abnormally high Stokes' shifted fluorescence of organic molecules. The main requirement of this process is the presence of intramolecular hydrogen bonding between the acidic and the basic moieties of the molecule in the ground state. Upon excitation to the first singlet state, the charge densities on these centers are changed, thereby drastically altering the acidic and basic properties of these groups and thus leading to intramolecular proton transfer. The acidic groups are in general $-\mathrm{OH}$ and $\mathrm{NH}_{2}$, and the basic groups are $=\mathrm{N}-$ and $>\mathrm{C}=\mathrm{O}$. Thus phototautomerizm could be keto-enol or amine-imine tautomerization [1].

Recently, we have found the existence of ESIPT in Nprotonated 2-amino-3-(2'-benzothiazolyl)quinoline cation in ethanol [2]. The investigation of ESIPT in this cation has shown that the fluorescence quantum yield and fluorescence lifetime of the hydrogen bonded form without the proton transfer (A) in ethanol increase essentially as the temperature decreases (the solvent viscosity increases). The fluorescence characteristics of the hydrogen bonded form with proton transfer (B) are changed insignificantly at the temperature decreasing. It points out that some excited-state deactivation process existing in form $A$ is inhibited at the temperature decreasing. It is interesting to elucidate the nature of this deactivation process and to establish the regularities of this process. The study of fluorescence behavior of nonprotonated aminoquinolines of similar structure may help to solve this problem. For this purpose at the present paper, such investigation was made for four derivatives of related aminoquinolines. The absorption and fluorescence spectra, the fluorescence quantum yields and fluorescence lifetimes and of 2-amino-3-(2'-benzothiazolyl)quinoline (I), 2-amino-3-(2'benzothiazolyl)quinoline (II), 2-amino-3-(2'-methoxybenzothiazolyl)quinoline (III), 2 -amino-3-( $2^{\prime}$-benzothiazolyl)benzothiazolyl (IV) at different temperatures in ethanol have been measured.

\section{EXPERIMENTAL}

2-amino-3-(2'-benzoxazolyl)quinoline (I), 2-amino-3-(2'benzothiazolyl)quinoline (II), 2 -amino-3-( $2^{\prime}$-methoxybenzothiazolyl)quinoline (III), 2-amino-3-(2'-benzothiazolyl)benzoquinoline (IV) were kindly provided by Professor E. Daltrozzo (Konstanz University, Germany). Solvents usedheptane, dioxane, toluene, tetrahydrofuran, ethylacetate, acetone, ethanol, acetonitrile, butyronitrile, dimethylformamide, glycerol, and dimethylsulfoxide - were purified according to methods known [3]. The absorption spectra were registered by Shimadzu UV-3100 spectrophotometer, and the fluorescence spectra by Elyumin 2M spectrofluorimeter. The fluorescence quantum yields were measured by comparison of the areas under the corrected fluorescence spectra of 
<smiles>c1ccc2c(c1)NNNc1[nH]c3ccccc3c1S2</smiles>

A<smiles>Nc1nc2ccccc2cc1-c1nc2ccccc2o1</smiles>

I<smiles>COc1ccc2nc(-c3cc4ccccc4nc3N)sc2c1</smiles>

III<smiles></smiles>

B<smiles>Nc1nc2ccccc2cc1-c1nc2ccccc2s1</smiles>

II<smiles>Nc1nc2cc3ccccc3cc2cc1-c1nc2ccccc2s1</smiles>

IV the solutions of the compounds investigated in proper solvent and quinine bisulphate in $1 \mathrm{~N}$ sulphuric acid $\left(\varphi_{f}=\right.$ 0.546) [4]. The optical densities of the solutions investigated were in the range $0.2-0.4$ at the excitation wavelength. The fluorescence quantum yields at different temperatures were corrected with respect to the absorption spectra of solutions at the same temperatures. The optical cryostat with liquid nitrogen vapor cooling was used for absorption and fluorescence spectral and kinetic measurements in the range $77-295 \mathrm{~K}$. The fluorescence kinetics was registered by SP-70 nanoseconds spectrometer by the method of time-correlated counting of single photons with excitation by air-filled flash lamp radiation (excitation pulse duration $0.8 \mathrm{~ns}$, registration channel width $0.054 \mathrm{~ns}$ ). The decay times were fitted using the iterative deconvolution procedure which allowed a time resolution down to $0.1 \mathrm{~ns}$ and an accuracy of the fluorescence lifetime determination not more than $0.3 \mathrm{~ns}$. The fluorescence rate constants $k_{f}$ have been calculated by Strickler-Berg formula [5] to compare them with experimental data

$$
k_{f}=2.88 \times 10^{-9} n^{2} \frac{\int I(\tilde{v}) d \widetilde{v}}{\int I(\widetilde{v}) \widetilde{v}^{-3} d \widetilde{v}} \int \frac{\varepsilon(\widetilde{v})}{\widetilde{v}} d \widetilde{v},
$$

where $n$ is the solvent refraction index, $I$ is the fluorescence intensity, and $\varepsilon$ is the extinction coefficient of the absorption.

The conformation analysis was carried out by AM1, dipole moments in the ground and excited-states were calculated by AM1 (HyperChem ${ }^{\mathrm{TM}}$, Release 7.52 for Windows, Hypercube, Inc.). The dipole moments in the excited-state were determined by Bakhshiev's method [6] taking into consideration the change of dipole moment direction at the excitation.

$$
\begin{aligned}
\left(\widetilde{v}_{\text {sol }}^{8}-\tilde{v}_{\text {vap }}^{8}\right) & \\
= & C_{2}\left[\left(\frac{\varepsilon-1}{\varepsilon+2}\right)\left(\frac{2 n_{0}^{2}+1}{n_{0}^{2}+2}\right)^{2}\right. \\
& \left.\quad-\left(\frac{\varepsilon-1}{\varepsilon+2}-\frac{n_{0}^{2}-1}{n_{0}^{2}+2}\right)^{2}\left(\frac{2 n_{0}^{2}+1}{n_{0}^{2}+2}\right)\left(\frac{n_{0}^{2}-1}{n_{0}^{2}+2}\right)\right] \\
+ & C_{3} \frac{n_{D}^{2}-1}{2 n_{D}^{2}+1}+C_{4} \frac{n_{D}^{2}-1}{n_{D}^{2}+2}, \\
\Delta \widetilde{v}^{a-f}= & \text { const }+\Delta C^{a-f}\left(\frac{\varepsilon-1}{\varepsilon+2}-\frac{n_{0}^{2}-1}{n_{0}^{2}+2}\right)\left(\frac{2 n_{0}^{2}+1}{n_{0}^{2}+2}\right)^{2},
\end{aligned}
$$


TABLE 1: Dipole moments of compounds I-IV in the ground $\left(\mu_{g}\right)$, excited $\left(\mu_{e}\right)$ states, and the angle between them $(\alpha)$.

\begin{tabular}{cccccc}
\hline Compound & \multicolumn{1}{c}{ Method AM1 } & \multicolumn{2}{c}{ Bakhshiev's method } \\
& $\mu_{g}, \mathrm{D}$ & $\mu_{e}, \mathrm{D}$ & $\alpha$, degrees & $\mu_{e}, \mathrm{D}$ & 3.3 \\
I & 1.9 & 2.9 & 43 & 3.4 & 75 \\
II & 2.2 & 1.6 & 24 & 2.5 & 120 \\
III & 1.5 & 1.4 & 47 & 4.5 & 100 \\
IV & 2.4 & 1 & 122 & $\mu_{2}$ \\
\hline
\end{tabular}

TABLE 2: The maxima of absorption $\left(\lambda_{a}\right)$ and fluorescence $\left(\lambda_{f}\right)$ spectra, fluorescence quantum yields $\left(\varphi_{f}\right)$ and lifetimes $\left(\tau_{f}\right)$, and the fluorescence rate constants $\left(k_{f}\right)$ of $\mathbf{I}-\mathbf{I V}$ in ethanol at $295 \mathrm{~K}$ and $77 \mathrm{~K}$.

\begin{tabular}{clllll}
\hline Compound & $\lambda_{a},(\mathrm{~nm})$ & $\lambda_{f},(\mathrm{~nm})$ & $\varphi_{f} \cdot 10^{2}$ & $\tau_{f},(\mathrm{~ns})$ & $1.7(7.0)$ \\
\hline I & $400(407)$ & $455(430)$ & $9.8(92)$ & $0.9(2.1)$ & $5.8(13.1)$ \\
II & $406(414)$ & $469(446)$ & $0.17(1.5)$ & $1.2(2.7)$ & $0.18(0.7)$ \\
III & $405(415)$ & $457(439)$ & $0.25(6)$ & $0.2(2.3)$ \\
IV & $450(458)$ & $574(525)$ & $0.26(13.5)$ & $1.1(20.9)$ & $0.2(0.65)$ \\
\hline
\end{tabular}

here, $\widetilde{v}^{8}=\left(\widetilde{v}^{a}+\widetilde{v}^{f}\right) / 2, \Delta \widetilde{v}^{a-f}$ denotes fluorescence Stokes' shift, $\varepsilon$ denotes solvent dielectric constant, and $n$ denotes solvent refraction index. The parameters $C_{2}, \Delta C^{a-f}$ are equal,

$$
\begin{gathered}
\Delta C^{a-f}=\frac{2}{h c r^{3}}\left(\mu_{g}^{2}+\mu_{e}^{2}-2 \mu_{g} \mu_{e} \cos \alpha\right), \\
C_{2}=\frac{\mu_{g}^{2}-\mu_{e}^{2}}{h c r^{3}}
\end{gathered}
$$

here, $h$ denotes Planck's constant, $c$ denotes the light velocity, $r$ denotes Onsager's radius, $\mu_{g}, \mu_{e}$ denote the dipole moments at the ground and excited-states, and $\alpha$ denotes the angle between $\mu_{g}$ and $\mu_{e}$,

$$
\begin{gathered}
\mu_{e}=\sqrt{\mu_{g}-C_{2} h c r^{3}} \\
\cos \alpha=\frac{1}{2 \mu_{g} \mu_{e}}\left[\left(\mu_{g}^{2}+\mu_{e}^{2}\right)-\frac{\Delta C^{a-f}}{2 C_{2}}\left(\mu_{g}^{2}-\mu_{e}^{2}\right)\right] .
\end{gathered}
$$

\section{RESULTS AND DISCUSSION}

The absorption spectra of I-IV do not depend on the solvent polarity, but the fluorescence spectra of these compounds in various solvents are shifted to longwavelength region as the solvent dielectric constant increases. It points out that the solvent orientational relaxation takes place because of charge redistribution in excited fluorophor molecule. $\mu_{g}, \mu_{e}$, and $\alpha$ calculated by method AM1 and determined from spectral data by Bakhshiev's method are given in Table 1.

It is seen from Table 1 that the values of $\mu_{e}$ from spectral data are higher than those calculated by AM1 method. It can be caused by the existence of other excited-state relaxation processes (structural relaxation) resulting in the additional spectral displacement and higher $\mu_{e}$ values determined by Bakhshiev's method.

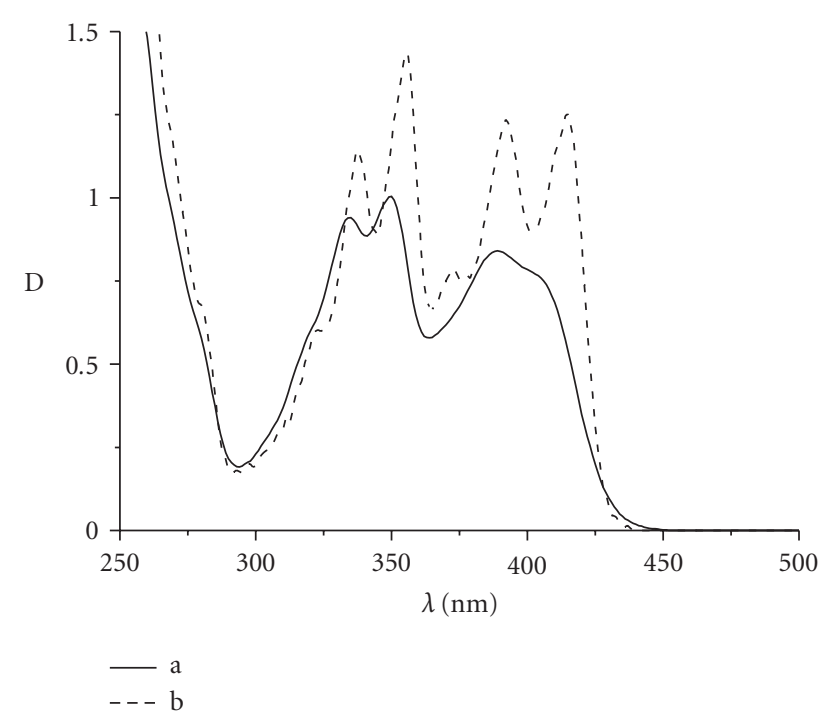

FIGURE 1: The absorption spectra of III in ethanol at $295 \mathrm{~K}$ (a) and 77 K (b).

The measurements of spectral characteristics at low temperatures give the additional information on the excited relaxation processes. The absorption spectra of compounds IIV in ethanol are shifted to longwavelength region as the temperature decreases. (Figure 1, Table 2). Similar results have been obtained for III in butyronitrile and methylcyclohexane. The shift of absorption spectra for these temperatures is equal to $8-10 \mathrm{~nm}$. Similar effect was obtained early for 2-pyridyl-5-aryloxazoles in ethanol [7].

The fluorescence spectra of I-IV (Figures 2-5) are shifted to shortwavelength region and fluorescence quantum yield increases as the temperature of the ethanol solution decreases. It points out that the excited-state relaxation process 


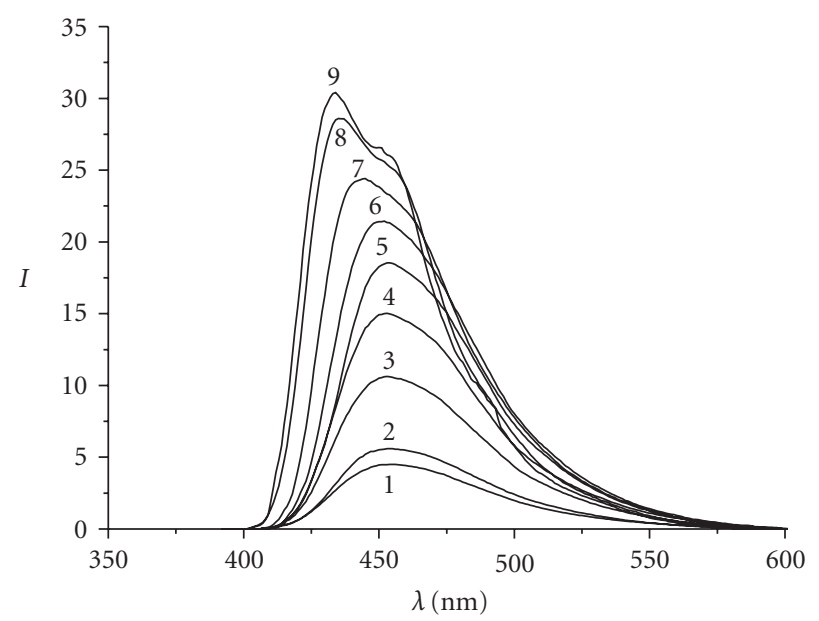
1. $295 \mathrm{~K}$
6. $175 \mathrm{~K}$
2. $273 \mathrm{~K}$
7. $150 \mathrm{~K}$
3. $250 \mathrm{~K}$
8. $130 \mathrm{~K}$
$4.225 \mathrm{~K}$
9. $115 \mathrm{~K}$
5. $200 \mathrm{~K}$

Figure 2: The fluorescence spectra of I in ethanol at different temperatures.

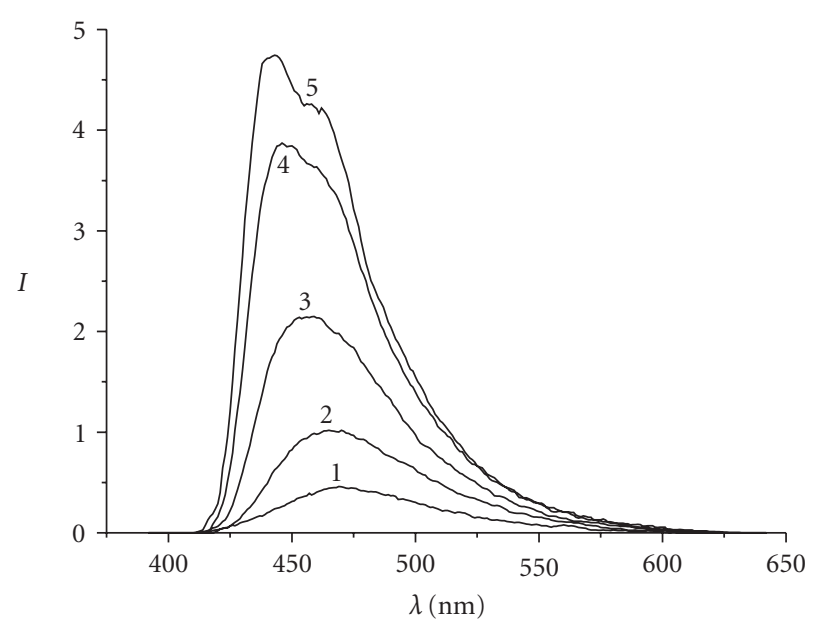
1. $295 \mathrm{~K}$
4. $115 \mathrm{~K}$
2. $200 \mathrm{~K}$
$5.77 \mathrm{~K}$
3. $150 \mathrm{~K}$

FIgURE 3: The fluorescence spectra of II in ethanol at different temperatures.

takes place. The rate of this process is determined by the medium properties, changing at the decreasing of the temperature. It is known that the viscosity [8] and polarity [9] of ethanol increase as the temperature decreases. If the position of the fluorescence spectra of compounds I-IV is determined only by the solvent polarity, the longwavelength shift of the fluorescence spectrum should be observed at the temperature decreasing. Therefore the observed shortwave-

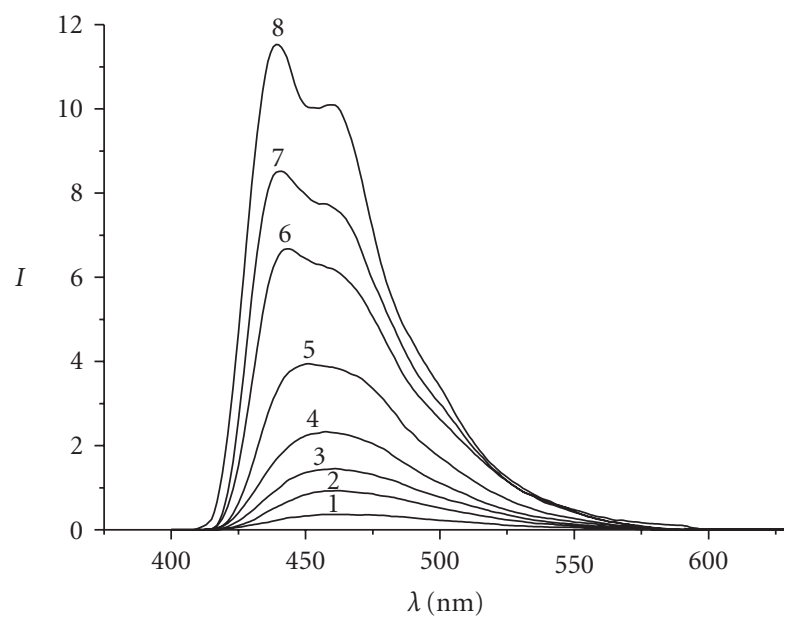
1. $295 \mathrm{~K}$
5. $150 \mathrm{~K}$
2. $225 \mathrm{~K}$
6. $130 \mathrm{~K}$
3. $200 \mathrm{~K}$
7. $115 \mathrm{~K}$
4. $175 \mathrm{~K}$
$8.77 \mathrm{~K}$

FIgure 4: The fluorescence spectra of III in ethanol at different temperatures.

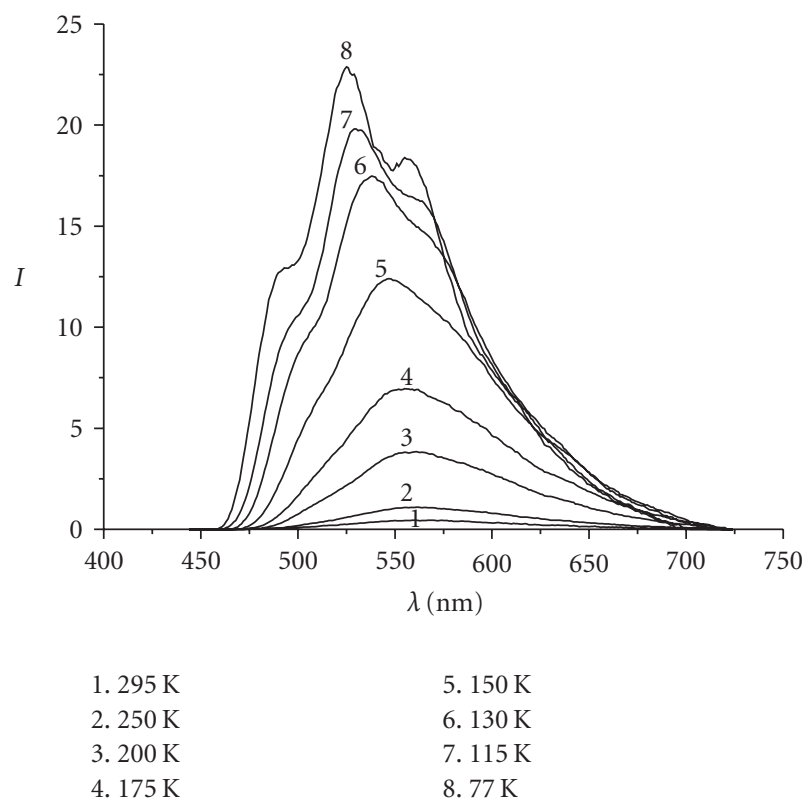

FIGURE 5: The fluorescence spectra of IV in ethanol at different temperatures.

length shift of fluorescence spectra of compounds studied at the temperature decreasing is caused by the increasing of the ethanol viscosity but not the solvent polarity.

The shortwavelength shift of fluorescence spectra of IIV at the temperature decreasing (at the increasing of the solvent viscosity) points out that the rate of the relaxation process depends essentially on the viscosity. Therefore, it can be concluded that this process is caused either by 


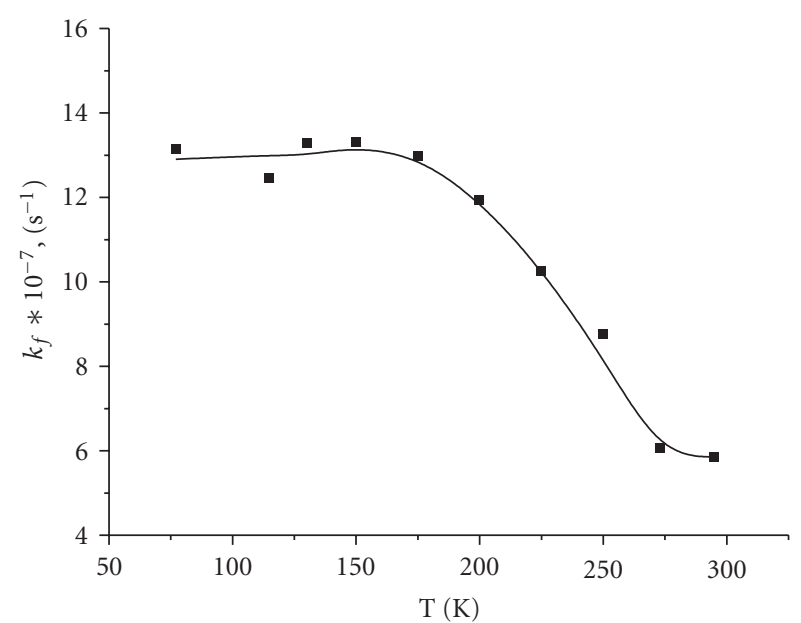

(a)

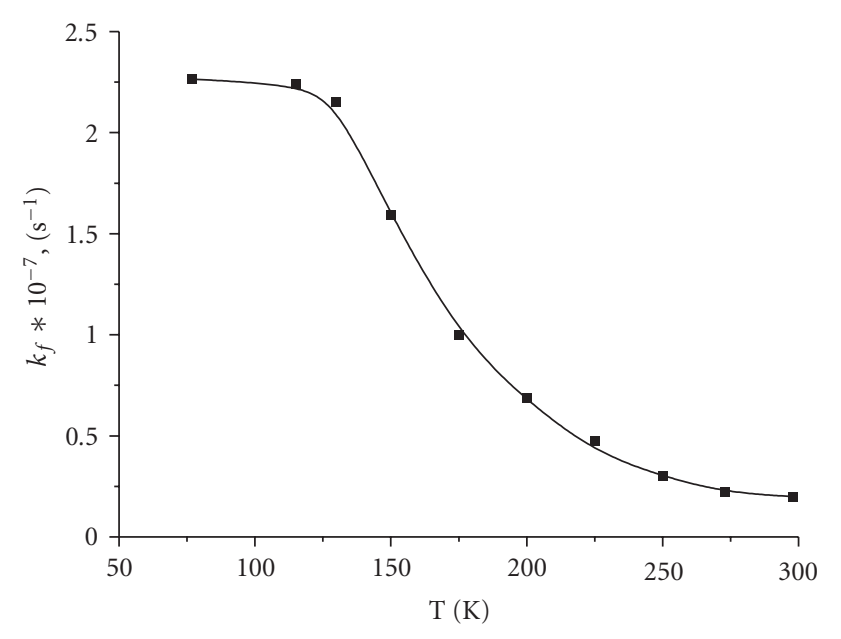

(c)

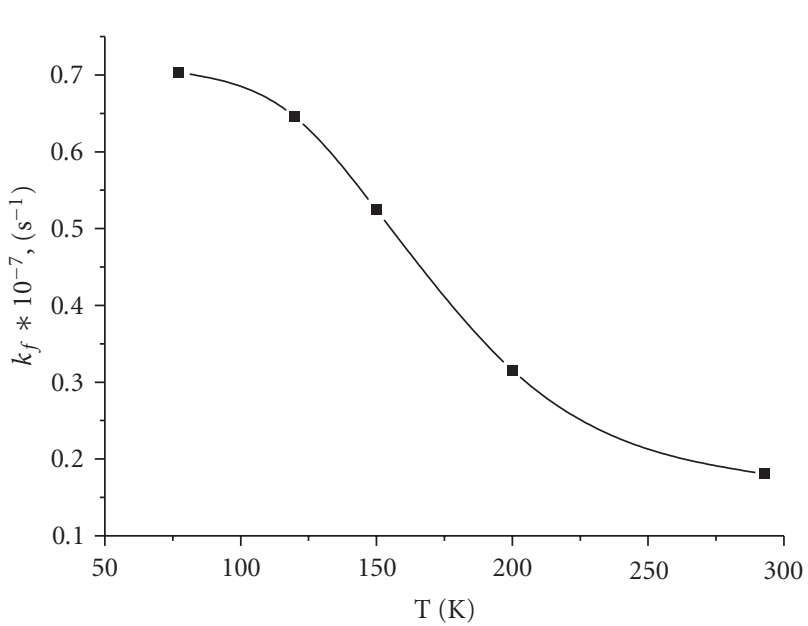

(b)

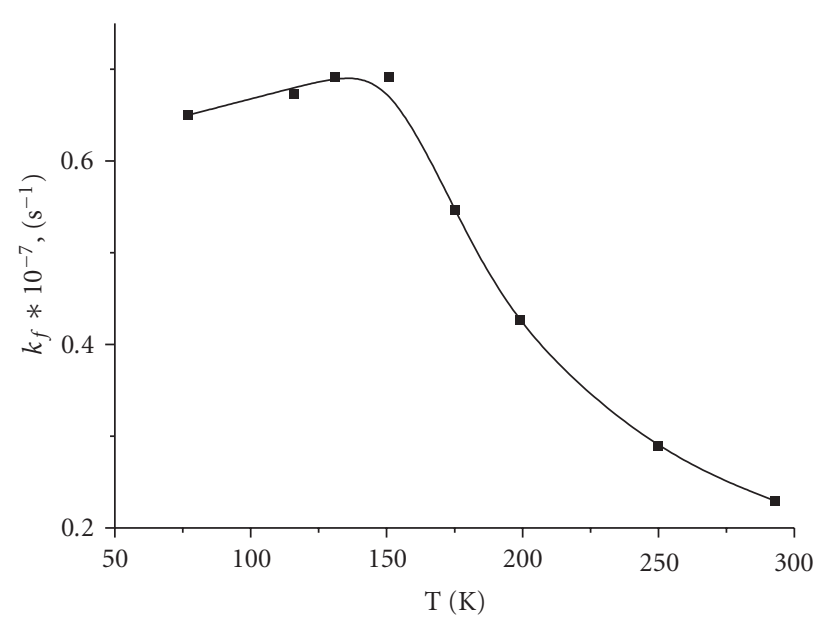

(d)

FIGURE 6: The dependence of the effective radiative rate constant on the temperature in ethanol for I(a), II(b), III(c), IV(d) compounds.

intramolecular mutual rotation of the molecular fragments of the fluorophor, or by orientational relaxation of the solvent molecules.

The fluorescence spectrum shift to shortwavelength region at the temperature decreasing is the consequence of the inhibition of the solvent orientational relaxation, resulting from the change of the dipole moment of fluorophor molecule at the excitation.

The dependence of the fluorescence rate constant $k_{f}$ on the temperature can give an important information about the relaxation process mechanism. If the relaxation process is associated with a mutual rotation of fluorophor molecular fragments, resulting in another molecular structure, then $k_{f}$ of relaxed and nonrelaxed states should differ significantly [10]. At the solvent relaxation, the molecular structure is changed insignificantly; therefore in this case $k_{f}$ of relaxed and nonrelaxed states should not differ considerably. We have determined the excited-state lifetimes and fluores- cence quantum yields at corresponding temperatures for $k_{f}$ calculation, $k_{f}=\varphi_{f} / \tau_{f}$. The fluorescence kinetics was registered at the wavelength which corresponds to the fluorescence spectral maximum at a given temperature. The dependence of $k_{f}$ on the temperature for I-IV is given in Figure 6.

The existence of structural relaxation is also supported by the great difference of $k_{f}^{77} / k_{f}^{298}$ calculated by StricklerBerg formula and this value, determined from the experimental data $\varphi_{f}$ and $\tau_{f}$. The first one is equal to 1.4, the second one is equal to 3.9. The quantum chemistry calculations give additional support of structural relaxation. The analysis of conformations with different torsion angles between quinolyl and thiazolyl (oxazolyl) rings has shown that in the ground-state nonplanar conformations with the angles 23, 58, 59, 63 degrees are most favourable for I, II, III, IV, respectively, but in the excited-state, planar ones are. It is interesting that the least value of $k_{f}^{77} / k_{f}^{298}$ and the least rotation angle for I correspond to the best agreement of excited-state 
dipole moments determined from the spectral data and calculated by AM1 method.

The values of $k_{f}$ at intermediate temperatures are effective ones and they have no physical meaning. The essential difference of $k_{f}$ for relaxed (final) and nonrelaxed (initial) forms proves the existence of excited-state structural relaxation in aminoquinolines studied consisting in the mutual rotation of quinolyl and thiazolyl (oxazolyl) fragments followed by the solvent orientation relaxation. We believe that such processes can be valid for the form without proton transfer in N-protonated 2-amino-3( 2 '-benzothiazolyl)quinoline cation [2] and can cause the fluorescence quantum yield increasing of this form at the temperature decreasing.

\section{CONCLUSION}

The data resulting from the investigation of fluorescence spectra and fluorescence kinetics of 2-amino-3-(2' -benzoxazolyl)quinoline, 2-amino-3-(2'-benzothiazolyl)quinoline, 2-amino-3-(2'-methoxybenzothiazolyl)quinoline, and 2amino-3-(2'-benzothiazolyl)benzoquinoline in ethanol at different temperatures show the existence of excited-state structural relaxation consisting in the mutual rotation of quinolyl and thiazolyl (oxazolyl) fragments followed by the solvent orientation relaxation. The rates of these processes are determined by the temperature-dependent viscosity of the medium.

\section{ACKNOWLEDGMENTS}

The authors thank Professor E. Daltrozzo (Konstanz University, Germany) for placing the substances I-IV at their disposal. The support of the Russian Foundation for Basic Researches (grant 03-03-32687) is gratefully acknowledged.

\section{REFERENCES}

[1] S. J. Formosinho and L. G. Arnaut, "Excited-state proton transfer reactions II. Intramolecular reactions," Journal of Photochemistry and Photobiology A: Chemistry, vol. 75, no. 1, pp. 21-48, 1993.

[2] F. Menges, E. Daltrozzo, M. Kammerer, Yu. A. Mednykh, and B. M. Uzhinov, in XIX IUPAC Symposium on Photochemistry, p. 336, Budapest, Hungary, July 2002, Abstr.

[3] A. Weissberger, E. S. Proskauer, J. A. Riddick, and E. E. Toops, Organic Solvents. Physical Properties and Methods of Purification, vol. 7 of Technique of Organic Chemistry, Wiley Interscience, New York, NY, USA, 1955.

[4] W. Melhuish, The Journal of Physical Chemistry A, vol. 76, p. 547, 1972.

[5] S. J. Strickler and R. A. Berg, "Relationship between absorption intensity and fluorescence lifetime of molecules," The Journal of Chemical Physics, vol. 37, no. 4, pp. 814-822, 1962.

[6] J. R. Lakowicz, Principles of Fluorescence Spectroscopy, Plenum Press, New York, NY, USA, 1983.

[7] M. N. Khimich, V. V. Volchkov, and B. M. Uzhinov, "Fluorescence study of excited-state relaxation processes of 2-pyridyl5-aryloxazoles," Journal of Fluorescence, vol. 13, no. 4, pp. 301305, 2003.
[8] T. K. Sherwood, R. C. Reid, and J. M. Prausnitz, The Properties of Gases and Liquids, McGraw-Hill, New York, NY, USA, 1977.

[9] C. P. Smyth and W. N. Stoops, "The dielectric polarization of liquids. VI. Ethyl iodide, ethanol, normal-butanol and normal-octanol," Journal of the American Chemical Society, vol. 51, no. 11, pp. 3312-3329, 1929.

[10] M. N. Khimich, N. I. Makarova, M. I. Knyazhansky, and B. M. Uzhinov, "Excited state structural relaxation of N-(1-anthryl)2,4,6-trimethyl-pyridinium cation," International Journal of Photoenergy, vol. 6, no. 2, pp. 69-72, 2004. 


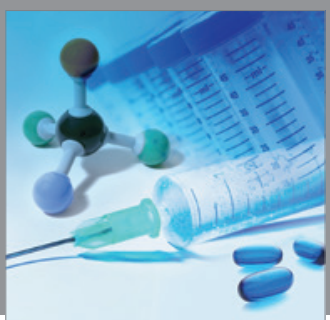

International Journal of

Medicinal Chemistry

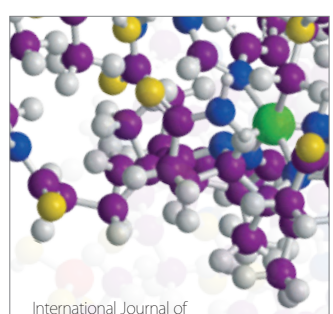

Carbohydrate Chemistry

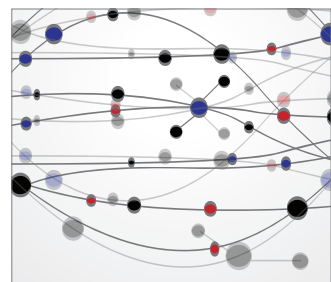

The Scientific World Journal
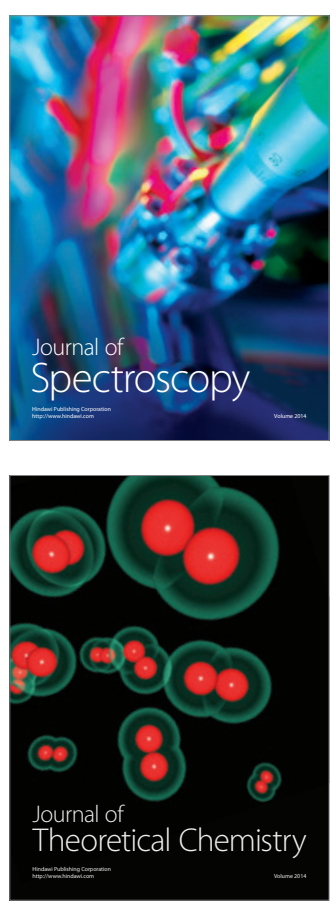
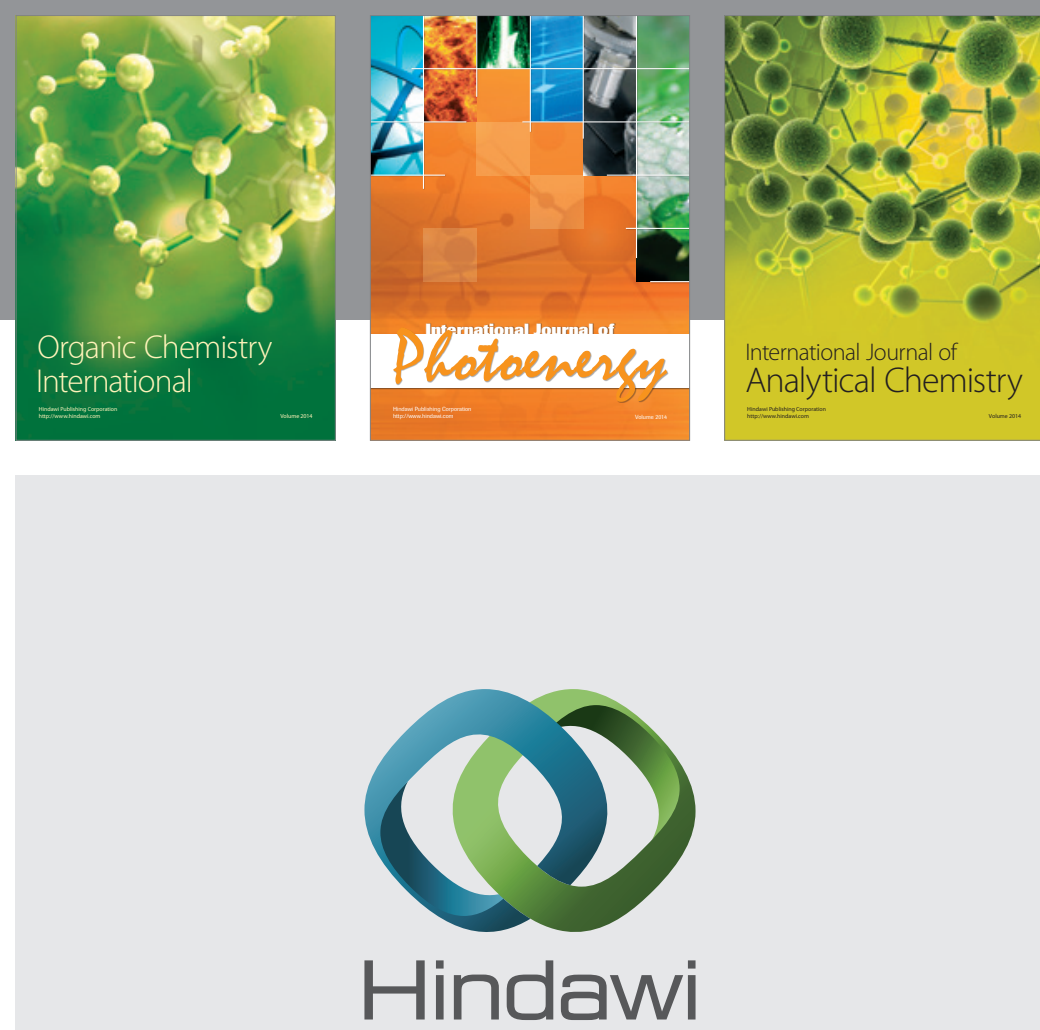

Submit your manuscripts at

http://www.hindawi.com
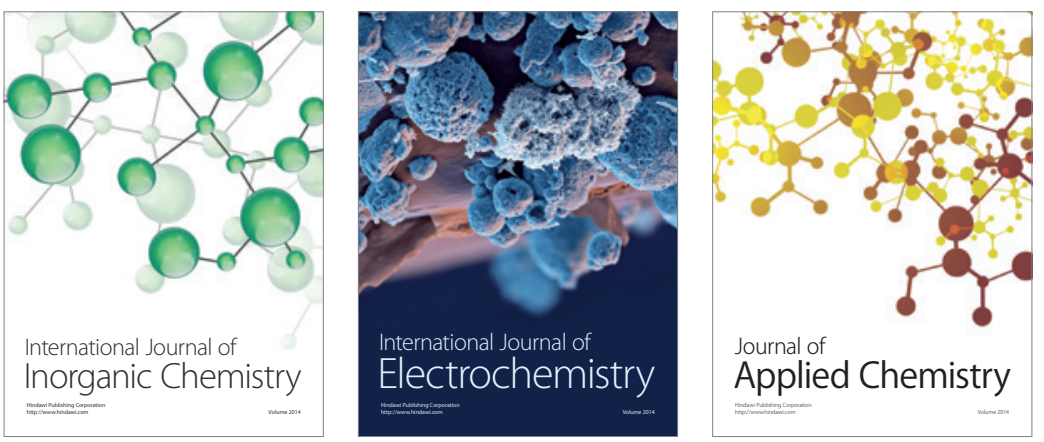

Journal of

Applied Chemistry
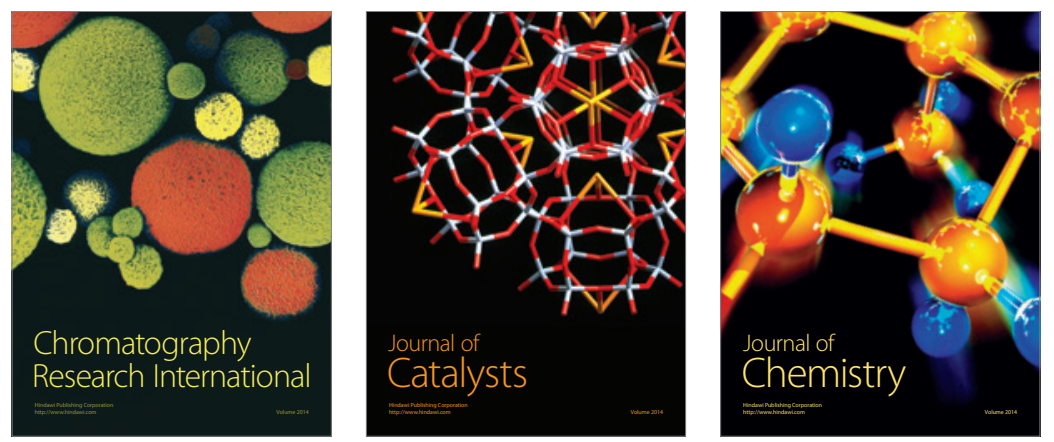
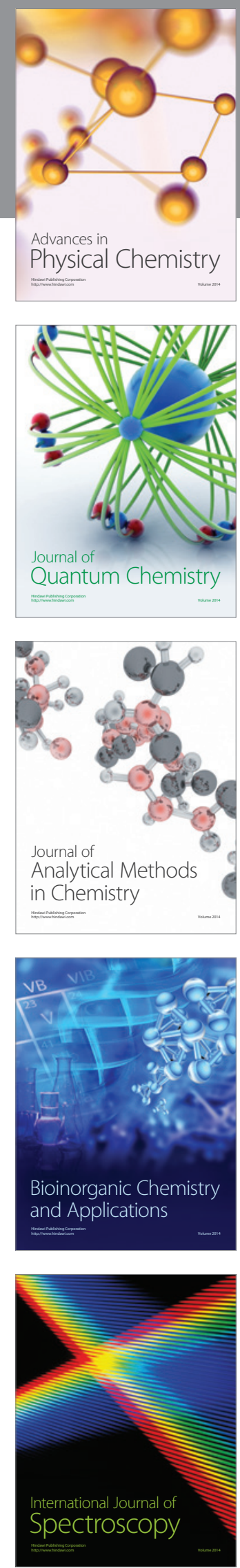